\title{
Long non-coding RNA UCA1 is a predictive biomarker of cancer
}

\author{
Han-han Hong ${ }^{1, *}$, Li-kun Hou ${ }^{2, *}$, Xin Pan ${ }^{3, *}$, Chun-yan $\mathrm{Wu}^{2}$, Hai Huang ${ }^{1}$, Bing Li ${ }^{1}$, Wei Nie ${ }^{1}$ \\ ${ }^{1}$ Department of Respiratory Medicine, Shanghai Changzheng Hospital, Second Military Medical University, Shanghai, China \\ ${ }^{2}$ Department of Pathology, Shanghai Pulmonary Hospital, Tongji University School of Medicine, Shanghai, China \\ ${ }^{3}$ Department of Medical Section, Zhenjiang Emergency Medical Center, Zhenjiang, Jiangsu, China \\ *These authors contributed equally to this work
}

Correspondence to: Chun-yan Wu, email: chunyan_wu2015@126.com

Hai Huang, email: haihuang7207@163.com

Bing Li, email: bing_li1962@163.com

Wei Nie, email: niewei-1001@163.com

Keywords: cancer, UCA1, biomarker

Received: March 22, $2016 \quad$ Accepted: June 03, $2016 \quad$ Published: June 17, 2016

\section{ABSTRACT}

Human urothelial carcinoma associated 1 (UCA1) is a long noncoding RNA that is putatively oncogenic in solid tumors. This meta-analysis investigated an association between UCA1 levels and survival times of cancer patients. The primary endpoints were overall survival (OS) and progression-free survival (PFS). A comprehensive, computerized literature search was conducted of the databases PubMed, EMBASE, Chinese National Knowledge Infrastructure (CNKI), and Wanfang. The strength of association between UCA1 and cancer prognosis was assessed by computing the hazard ratio (HR) with its corresponding $95 \%$ confidence interval (CI). Twelve studies comprising 954 cancer patients met the criteria for this meta-analysis. Overall, a significant negative association was found between UCA1 levels and OS time (HR1.81, 95\% CI1.52-2.17), including the following cancers analyzed independently: colorectal (HR2.61, 95\% CI1.56-4.37), non-small cell lung (HR1.49, 95\% CI1.16-1.90), gastric (HR2.19, 95\% CI1.36-3.51), and ovarian (HR1.89, 95\% CI1.14-3.12). There was also a significant negative association between UCA1 levels and PFS time (HR2.59, 95\% CI1.61-4.16). In conclusion, this meta-analysis indicated that higher levels of UCA1 correlate with shorter PFS and OS times in cancers.

\section{INTRODUCTION}

Cancer is a major public health problem worldwide, with overall death rates that rose during most of the 20 th century. In the United States, cancer is the second leading cause of death [1]. In China, cancers are the leading cause of death, despite the development of effective drugs and supportive care [2].

Long noncoding RNAs (lncRNAs) are non-proteincoding molecules, longer than 200 nucleotides [3]. Many studies have reported that IncRNAs are deregulated in cancers, suggesting that the aberrant expression of lncRNAs is associated with tumorigenesis, metastasis, and prognosis in cancer.

Human UCA1 (urothelial carcinoma associated 1) is a lncRNA that was first identified in human bladder carcinoma [4], and whose oncogenic effect may be related to glucose metabolism [5]. Recently, some studies have reported the relevance of $U C A 1$ in cancer prognosis and the acquired resistance to drugs [6-17]. For example, patients with advanced non-small cell lung cancer (NSCLC) that harbor mutations that activate epidermal growth factor receptor $(E G F R)$ can be treated with $E G F R$-tyrosine kinase inhibitors (TKIs) such as gefitinib. However resistance to this treatment is often acquired, most commonly via a secondary $T 790 M$ mutation. Cheng et al. found that the IncRNA UCAI was upregulated in resistant cells, and that overexpression of UCA1 was associated with shorter progression-free survival (PFS) in non-resistant cells [8]. Furthermore, UCA1 knockdown restored sensitivity to gefitinib in acquired-resistant NSCLC cells without the T790M mutation, and inhibited the activation of the AKT/mTOR pathway and epithelialmesenchymal transition.

No meta-analysis was been conducted to assess the association between $U C A 1$ and the survival of patients 
with cancers. Therefore, this meta-analysis investigated an association between UCAI and the survival of cancer patients. Overall survival (OS) and PFS were the primary endpoints.

\section{RESULTS}

\section{Study characteristics}

The initial search of the databases produced 53 studies (Figure 1). After excluding duplicate articles, 49 potentially eligible studies were selected. After a detailed evaluation, 12 studies were selected for the final metaanalysis with a total of 954 cancer patients (Table 1). Of the 12 studies, 2, 3, 2, and 2 concerned colorectal cancer, NSCLC, ovarian cancer, and gastric cancer, respectively, and there was one study each regarding esophageal squamous cell carcinoma and hepatocellular carcinoma.

Not all studies examined both OS and PFS, because most of the studies were retrospective cohort studies; 10 studies investigated the association between UCA1 and OS, while 3 studies assessed the association between $U C A 1$ and PFS.

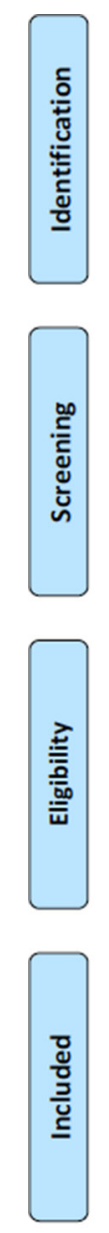

\section{Results of the meta-analysis}

The association between the expression of UCA1 and OS was investigated in 10 studies (Figure 2). We found a statistically significant negative association between levels of $U C A 1$ and $\mathrm{OS}(\mathrm{HR}=1.81,95 \% \mathrm{CI}=1.52-2.17)$. In a subgroup analysis of cancer sites, significant negative associations were found between levels of UCA1 and OS in the following cancers: colorectal (HR2.61, 95\% CI1.564.37), NSCLC (HR1.49, 95\% CI1.16-1.90), gastric cancer (HR2.19, 95\% CI1.36-3.51), and ovarian cancer (HR1.89, 95\% CI1.14-3.12). When the studies that adjusted for lymph node and clinical stage were included, shorter OS was also observed (HR1.71, 95\% CI $=1.42-2.07)$. We did not perform subgroup analyses for esophageal squamous cell carcinoma or hepatocellular carcinoma, because no more than one study each investigated these associations between UCA1 and OS.

The association between UCAI and PFS was investigated in 3 studies (Figure 3). There was a significant negative association between UCA1 levels and PFS (HR2.59, 95\% CI1.61-4.16; Figure 3). All the results are listed in the Table 2.

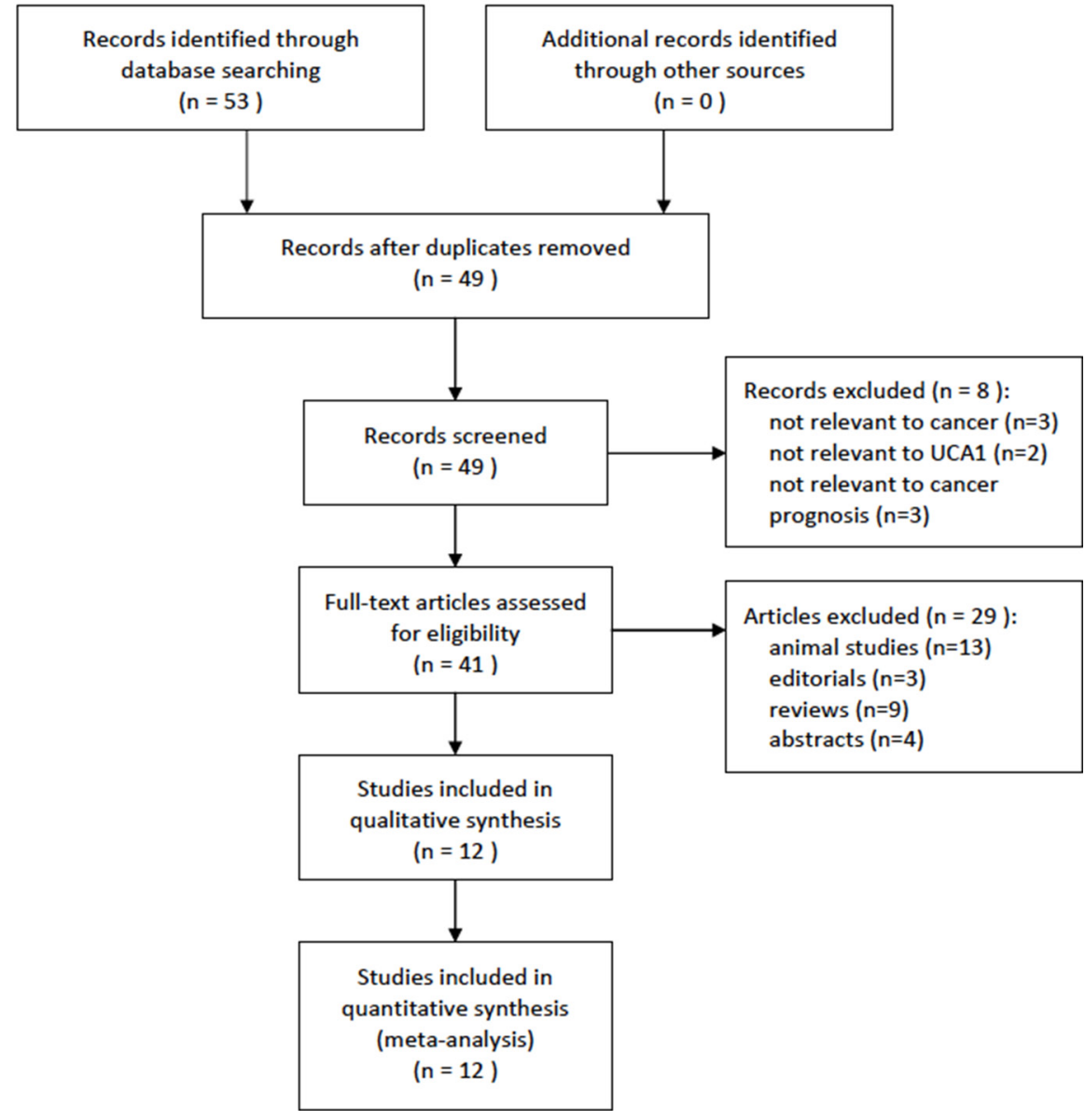

Figure 1: Flow of study selection. 
Table 1: Characteristics of the included studies ${ }^{\mathrm{a}}$

\begin{tabular}{|c|c|c|c|c|c|c|c|c|c|c|}
\hline $\begin{array}{l}\text { First } \\
\text { author }\end{array}$ & Year & $\mathbf{n}^{\mathbf{b}}$ & $\mathbf{A g e}^{\mathrm{c}}$ & Men, $\%$ & Reference & $\begin{array}{l}\text { FU, } \\
\text { mo }\end{array}$ & Cancer & Outcome & Co-variants & NOS \\
\hline Cheng & 2015 & 94 & NA* & 46.4 & GAPDH & 24 & NSCLC & PFS & Age & 7 \\
\hline Gao & 2015 & 20 & NA & NA & GAPDH & NA & $\mathrm{GC}$ & OS & Lymph node; clinical stage & 8 \\
\hline Han & 2014 & 80 & 55 & 49 & GAPDH & 42.6 & $\mathrm{CRC}$ & OS & NA & 8 \\
\hline Kamel & 2015 & 82 & 57 & 68.3 & GAPDH & NA & $\mathrm{HCC}$ & PFS & Barcelona clinic liver Cancer stage; Child score; mean tumor size & 8 \\
\hline $\mathrm{Li}$ & 2014 & 90 & 60 & 55.6 & GAPDH & 43 & ESCC & OS & Differentiation grade; lymph node; clinical stage & 7 \\
\hline $\mathrm{Ni}$ & 2015 & 54 & NA & 72.2 & GAPDH & NA & $\mathrm{CRC}$ & OS & $\begin{array}{l}\text { Lymphatic invasion; lymph node; distant metastasis; clinical } \\
\text { stage }\end{array}$ & 8 \\
\hline Nie & 2015 & 112 & 63.2 & 59.8 & GAPDH & 45 & NSCLC & OS & Lymph node; clinical stage & 8 \\
\hline Tao & 2015 & 80 & 65.1 & 60 & RUN6 & NA & $\mathrm{CRC}$ & $\mathrm{OS}$ & Lymph node; clinical stage & 7 \\
\hline Wang & 2015 & 60 & NA & 61.7 & GAPDH & NA & NSCLC & OS & Lymph node; clinical stage & 7 \\
\hline Yang & 2016 & 53 & NA & 0 & GAPDH & NA & Ovary & OS & Lymph node & 8 \\
\hline Zhang & 2016 & 117 & 33 & 0 & RUN6 & 22 & Ovary & OS & Chemotherapy response; lymph node; clinical stage & 8 \\
\hline Zheng & 2015 & 112 & NA & 57.1 & GAPDH & NA & $\mathrm{GC}$ & OS, PFS & $\begin{array}{l}\text { Tumor size; invasion depth; lymphatic metastasis; invade } \\
\text { adjacent organs; clinical stage }\end{array}$ & 8 \\
\hline
\end{tabular}

${ }^{a}$ This study characterized patients as $>65$ or $<65$; technique used to quantify UCA1 was real-time PCR in all studies; ${ }^{\mathrm{b}}$ sample size; ${ }^{\mathrm{c}}$ median age, y.

CRC, colorectal cancer; ESCC, esophageal squamous cell carcinoma; FU, follow-up; GAPDH, glyceraldehyde-3-phosphate dehydrogenase; GC, gastric cancer; HCC, hepatocellular carcinoma; NA, not available.

Study

ID

Han 2014
Li 2014
Gao 2015
Ni 2015
Nie 2015
Tao 2015
Wang 2015
Zheng 2015
Zhang 2016
Yang2016

Overall (I-squared $=19.1 \%, p=0.267$ )
$\mathrm{HR}(95 \% \mathrm{Cl})$

$4.68(1.36,16.14) \quad 2.06$

$2.63(1.42,4.87) \quad 8.31$

$2.02(1.02,4.00) \quad 6.77$

$3.10(1.17,8.22) \quad 3.32$

$1.41(1.08,1.84) \quad 44.45$

$2.00(1.00,4.00) \quad 6.57$

$1.94(1.06,3.55) \quad 8.64$

$2.35(1.22,4.53) \quad 7.35$

$1.69(1.00,2.86) \quad 11.47$

$6.32(1.12,35.66) \quad 1.05$

$1.81(1.52,2.17) \quad 100.00$

Figure 2: Meta-analysis for the association between $U C A 1$ and overall survival of cancer. 


\section{DISCUSSION}

This is the first meta-analysis to evaluate the association between UCA1 levels and cancer prognosis. We found that increased levels of $U C A 1$ were significantly associated with shorter OS and PFS times in cancer patients. In the subgroup analyses, UCA1 levels were significantly and negatively associated with OS times in colorectal cancer, NSCLC, ovarian cancer, and gastric cancer.

UCA1 putatively influences the proliferation, apoptosis, and cell cycle progression of colorectal cancer cells [6].Ni et al. [11]also found that knockdown of $U C A 1$ was associated with suppressed cell proliferation and metastasis in colorectal cancer cells. Nie et al. [12] suggested that silencing of UCAI impaired the proliferation and colony formation of NSCLC cells. Wang and coworkers [13] found that UCAllevels were associated with histological grade and lymph node metastasis in NSCLC. In addition, a clinicopathologic analysis revealed that $U C A 1$ levels correlated with worse differentiation, greater tumor size and invasion depth, and TNM stage in gastric cancer [14]. Thus, these data might explain why high levels of UCA1 were significantly associated with shorter OS and PFS in cancer patients in this meta-analysis.
The clinical implications of UCA1 in various cancers have not been studied well. Wang et al. [4] showed that a UCA1 assay was highly specific $(91.8 \%, 78$ of 85$)$ and very sensitive $(80.9 \%, 76$ of 94$)$ in the diagnosis of bladder cancer. However, Milowich et al. [18] indicated that the efficiency of the UCA1 test for detecting primary and recurring bladder cancer was low. Future studies should focus on the clinical utility of UCA1-based cancer diagnosis in clinical trials. In addition, $U C A 1$ has been implicated in the acquired resistance to EGFR-TKIs in EGFR-mutant NSCLC that did not include a T790M mutation [8]. Thus, the expression of UCA1 should be evaluated before patients receive EGFR-TKIs.

Some limitations of this meta-analysis should be pointed out. Firstly, the number of included studies in our meta-analysis was moderate. Secondly, most of the studies were conducted with Chinese sample populations and, therefore, our results may be applicable only to this ethnic group. Thirdly, not all of the studies reported the cutoff values of UCA1. Finally, many factors, such as gender and chemotherapy, may also affect OS and PFS. Thus, the results of this meta-analysis should be confirmed in future studies.

In conclusion, the results of this meta-analysis suggest that UCA1 may be a risk factor for shorter OS and

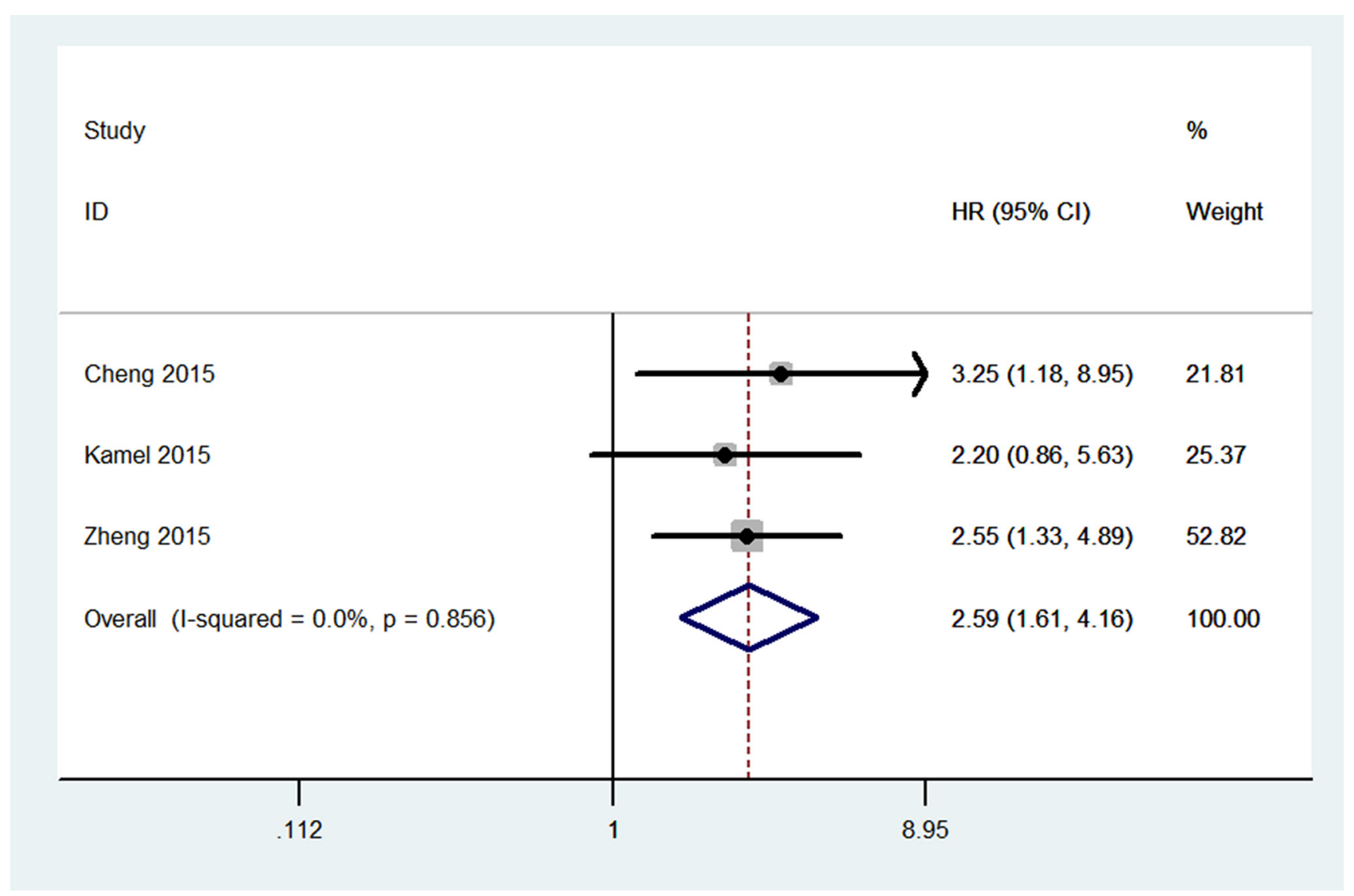

Figure 3: Meta-analysis for the association between $U C A 1$ and progression-free survival of cancer. 
Table 2: Results of this meta-analysis

\begin{tabular}{|l|c|c|c|c|c|}
\hline \multicolumn{2}{|c}{} & \multicolumn{3}{c}{ HR (95\% CI) } & \multicolumn{1}{c}{ I $^{2}(\%)$} \\
\hline Overall survival & & $1.81(1.52-2.17)$ & $<0.00001$ & 19 & 0.27 \\
\hline Site of cancer & CRC & $2.62(1.56-4.37)$ & 0.0002 & 0 & 0.46 \\
\hline & NSCLC & $1.49(1.16-1.90)$ & 0.001 & 0 & 0.34 \\
\hline & GC & $2.19(1.36-3.51)$ & 0.001 & 0 & 0.75 \\
\hline Adjusted lymph node and clinical stage & Ovary & $1.89(1.14-3.12)$ & 0.01 & 51 & 0.15 \\
\hline Progression-free survival & & $1.71(1.42-2.07)$ & $<0.00001$ & 0 & 0.43 \\
\hline
\end{tabular}

CRC, colorectal cancer; NSCLC, non-small cell lung cancer; GC, gastric cancer.

PFS in cancers. Well-designed studies with large sample sizes are needed to confirm further the association between $U C A 1$ and clinical outcomes of cancers in various ethnic populations.

\section{MATERIALS AND METHODS}

\section{Publication search}

We searched the databases PubMed, EMBASE, Chinese National Knowledge Infrastructure (CNKI) and Wanfang, to 14 March 2016, for relevant articles. The search terms used were "UCA1" and "cancer or carcinoma or tumor". Reference lists of relevant articles were also reviewed to identify potential eligible studies.

\section{Inclusion and exclusion criteria}

For inclusion in this meta-analysis, the studies met the following criteria: cohort design; investigated the association between $U C A 1$ and cancer prognosis (OS or PFS); and sufficient original data for calculating a hazard ratio (HR) with its $95 \%$ confidence interval (CI). A study was excluded if it was not relevant to cancer, UCA1, or cancer prognosis; involved animals; or was an editorial, review, or abstract. If more than one study used the same patient cases, the one with the most comprehensive population was included. Differences in opinion among the authors were solved by discussion.

\section{Data extraction and quality assessment}

Two investigators extracted and reviewed the data independently. The following data were extracted: the first author's name, year of publication, patient ages and genders, duration of follow-up, sample size, site of cancer, PFS, OS, and co-variants. Since all included studies were cohort studies, the Newcastle-Ottawa Scale (NOS) was used to evaluate the methodological quality [19].

\section{Statistical analysis}

The strength of association between UCA1 and cancer prognosis (PFS and OS) was assessed by computing the HR with its corresponding $95 \%$ CI. OS was defined as the time between diagnosis and death. PFS was defined as the time between diagnosis and progression. The heterogeneity among eligible studies was checked by using the chi-squared based $Q$-statistic test. The randomeffects model or fixed-effects model was used to analyze the pooled HRs. If the number of included studies in an analysis was more than 10, Egger's linear regression test and Begg's funnel-plot analysis were used to weigh the potential publication bias. All the $P$-values were determined by a 2-sided test. All statistical analyses were conducted using STATA software (version 12.0; Stata, College Station, Texas).

\section{ACKNOWLEDGMENTS AND FUNDING}

This work was supported by the National Natural Science Foundation of China (No.81172227), the Research Foundation of Shanghai Municipal Education Commission (No.12ZZ073), the Shanghai Natural Science Foundation (No.16ZR1428900), and the Shanghai Municipal Commission of Health and Family Planning (No. 201440398).

\section{CONFLICTS OF INTEREST}

None.

\section{REFERENCES}

1. Siegel RL, Miller KD, Jemal A. Cancer statistics, 2016. CA Cancer J Clin. 2016; 66:7-30.

2. Chen W, Zheng R, Baade PD, Zhang S, Zeng H, Bray F, Jemal A, Yu XQ, He J. Cancer statistics in China, 2015. CA Cancer J Clin. 2016. doi: 10.3322/caac.21338. [Epub ahead of print].

3. Kapranov P, Cheng J, Dike S, Nix DA, Duttagupta R, Willingham AT, Stadler PF, Hertel J, Hackermüller J, Hofacker IL, Bell I, Cheung E, Drenkow J, et al. RNA maps reveal new RNA classes and a possible function for pervasive transcription. Science. 2007; 316:1484-8.

4. Wang XS, Zhang Z, Wang HC, Cai JL, Xu QW, Li MQ, Chen YC, Qian XP, Lu TJ, Yu LZ, Zhang Y, Xin DQ, Na YQ, 
et al. Rapid identification of $U C A 1$ as a very sensitive and specific unique marker for human bladder carcinoma. Clin Cancer Res. 2006; 12:4851-8.

5. Li Z, Li X, Wu S, Xue M, Chen W. Long non-coding RNA $U C A 1$ promotes glycolysis by upregulating hexokinase 2 through the mTOR-STAT3/microRNA143 pathway. Cancer Sci. 2014; 105:951-5.

6. Han Y, Yang YN, Yuan HH, Zhang TT, Sui H, Wei XL, Liu L, Huang P, Zhang WJ, Bai YX. UCA1, a long noncoding RNA up-regulated in colorectal cancer influences cell proliferation, apoptosis and cell cycle distribution. Pathology. 2014; 46:396-401.

7. Li JY, Ma X, Zhang CB. Overexpression of long noncoding RNA UCA1 predicts a poor prognosis in patients with esophageal squamous cell carcinoma. Int J Clin Exp Pathol. 2014; 7:7938-44.

8. Cheng N, Cai W, Ren S, Li X, Wang Q, Pan H, Zhao M, Li J, Zhang Y, Zhao C, Chen X, Fei K, Zhou C, et al. Long non-coding RNA UCA1 induces non-T790M acquired resistance to EGFR-TKIs by activating the AKT/mTOR pathway in EGFR-mutant non-small cell lung cancer. Oncotarget. 2015; 6:23582-93.

9. Gao J, Cao R, Mu H. Long non-coding RNA UCA1 may be a novel diagnostic and predictive biomarker in plasma for early gastric cancer. Int J Clin Exp Pathol. 2015; 8:1293642.

10. Kamel MM, Matboli M, Sallam M, Montasser IF, Saad AS, El-Tawdi AH. Investigation of long noncoding RNAs expression profile as potential serum biomarkers in patients with hepatocellular carcinoma. Transl Res. 2016; 168:134 45.

11. Ni B, Yu X, Guo X, Fan X, Yang Z, Wu P, Yuan Z, Deng Y, Wang J, Chen D, Wang L. Increased urothelial cancer associated 1 is associated with tumor proliferation and metastasis and predicts poor prognosis in colorectal cancer. Int J Oncol. 2015; 47:1329-38.
12. Nie W, Ge HJ, Yang $X Q$, Sun $X$, Huang H, Tao X, Chen WS, Li B. LncRNA-UCA1 exerts oncogenic functions in non-small cell lung cancer by targeting miR-193a-3p. Cancer Lett. 2016; 371:99-106.

13. Wang HM, Lu JH, Chen WY, Gu AQ. Upregulated lncRNA-UCAl contributes to progression of lung cancer and is closely related to clinical diagnosis as a predictive biomarker in plasma. Int J Clin Exp Med. 2015; 8:1182430 .

14. Zheng Q, Wu F, Dai WY, Zheng DC, Zheng C, Ye H, Zhou B, Chen JJ, Chen P. Aberrant expression of UCA1 in gastric cancer and its clinical significance. Clin Transl Oncol. 2015; 17:640-6.

15. Zhang L, Cao X, Zhang L, Zhang X, Sheng H, Tao K. UCA1 overexpression predicts clinical outcome of patients with ovarian cancer receiving adjuvant chemotherapy. Cancer Chemother Pharmacol. 2016 Feb 6. [Epub ahead of print].

16. Tao K, Yang J, Hu Y, Sun Y, Tan Z, Duan J, Zhang F, Yan H, Deng A. Clinical significance of urothelial carcinoma associated 1 in colon cancer. Int J Clin Exp Med. 2015 Nov $15 ; 8: 21854-60$.

17. Yang Y, Jiang Y, Wan Y, Zhang L, Qiu J, Zhou S, Cheng W. $U C A 1$ functions as a competing endogenous RNA to suppress epithelial ovarian cancer metastasis. Tumour Biol. 2016 Feb 11. [Epub ahead of print]

18. Milowich D, Le Mercier M, De Neve N, Sandras F, Roumeguere T, Decaestecker C, Salmon I, Rorive S. Diagnostic value of the UCA1 test for bladder cancer detection: a clinical study. Springerplus. 2015; 4:349. doi: 10.1186/s40064-015-1092-6. eCollection 2015.

19. GA Wells, B Shea, D O'Connell, J Peterson, V Welch, M Losos, P Tugwell. The Newcastle-Ottawa Scale (NOS) for assessing the quality ofnonrandomised studies in metaanalyses. URL: http://www.ohri.ca/programs/clinical_ epidemiology/oxford.asp. 